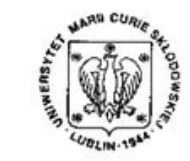

Annales UMCS Informatica AI X, 1 (2010) 87-92 DOI: $10.2478 / \mathrm{v} 10065-010-0039-3$

\section{Annales UMCS}

Informatica

Lublin-Polonia

Sectio AI

http://www.annales.umcs.lublin.pl/

\title{
Visualization of the extensive air shower and estimation of its parameters
}

\author{
Jan Malinowski*, Anna Śmiałkowska \\ Faculty of Physics and Applied Informatics, University of Lodz, \\ ul. Pomorska 149/153, 90-236 Eódz, Poland.
}

\begin{abstract}
In the process of cosmic ray penetrating the Earth's atmosphere new particles forming a cascade called the extensive air shower (EAS) are produced. The objective of our paper is to estimate chosen parameters and detailed visualization of the EAS based on the data received from simulations of physical processes describing this phenomenon. The simulation of the EAS is conducted using the CORSICA program. The tool presented in this paper allows estimating the altitude in the atmosphere where the EAS has the greatest number of particles, X_max, as well as the curvature radius of the produced particles on the front of the

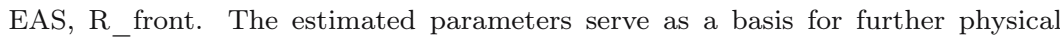
analysis and the visualization can serve for physicists as a tool for presentation and popularization of the described phenomena. The presented paper has an interdisciplinary character combining physics with IT. The program developed here is a tool for research in physics, in this case for the analysis of cosmic ray.
\end{abstract}

\section{Extensive Air Shower}

The EAS is a physical phenomenon initiated at the top of the atmosphere through high-energy particles coming from the outer space. Research on extensive showers is conducted in several large physical experiments, e.g. Auger [3], AGASA [4], Fly's Eye [5], and has an objective of explaining the mechanisms of cosmic ray occurrence and eventually learning about the rules of astrophysics.

*jmalinowski@uni.lodz.pl 


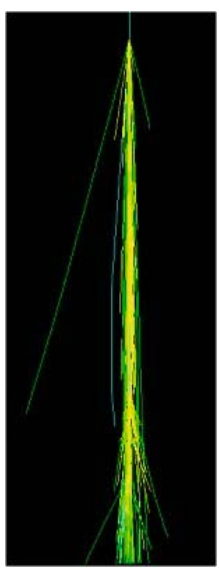

Fig. 1. The scheme of EAS

The source of the EAS are particles of high-energy cosmic ray, i.e. from $10^{12}$ to $10^{2} 0 \mathrm{eV}$. Entering the atmosphere, primary particles collide with the nuclei of the atmosphere atoms and produce secondary particles passing them their energy. Those produce further particles in next collisions. In this way, the cascade of particles is created. When the secondary particles start to lack energy, the cascade starts to expire, i.e. the number of produced particles declines. The EAS resulting from highenergy particles reaches its maximum from $10^{4}$ to $10^{10}$ particles approaching the Earth with the speed near that of light. The electrons of relatively low energies occur the most frequently. The depth in the atmosphere where the number of particles is the largest is $\mathrm{X} \_$max and is usually given in $\mathrm{g} / \mathrm{cm} 2$. The scheme illustrating the traces of the EAS particles received from simulations is depicted in Fig. 1.

\section{The Simulation of the EAS}

The simulation of the EAS is conducted using the CORSIKA [2] program. This tool makes a detailed simulation of physical processes occurring when the particles penetrate the atmosphere. Strong, weak and electromagnetic interactions of all particles are modelled here. As a result, we receive, e.g., data about the position in time and place of each created particle as well as about the end of its trajectory, i.e. the position of the next collision or the position where the particle stops.

\section{Data for the Visualization}

As an output of the CORSIKA program, among others, binary files containing the following information for each particle in the shower are received:

- the kind of particle and its energy,

- ts, xs, ys, zs - the position of the particle occurrence, i.e. the time and place of the beginning of the particle trace,

- te, xe, ye, ze - the position of the end of the particle trace.

Each particle is described by $40 \mathrm{~B}$ of data. The EAS has in the maximum of its development from about thousands to billions of particles. The sum of all particles in the shower can be around 100 times higher than the number of particles in the maximum. Thus describing the largest EAS involves using over $2000 \mathrm{~GB}$ of data. In 
such cases, detailed visualization is not realized because of both, the size of data and the overlapping of particles' traces. Such events are visualized only in an approximate way.

The objective of this paper is detailed visualization of the EAS for relatively small energies. One of the problems is the queuing of a large amount of data. Using dynamic structures directly for the visualization is not possible because of the long time needed for processing such an amount of data. We solve this problem with a two-step approach. Firstly, we conduct a preliminary file transformation preparing it for fast visualization. Secondly, the prepared data is read and depicted on the screen.

During the visualization of the EAS, we need information about the number of particles and their coordinates $\mathrm{x}, \mathrm{y}, \mathrm{z}$ for the given time intervals. Such data are received after the initial calculations. After being read from the binary file, they are placed on a dynamically created list. Each record of the list regards one particle and contains the data structure described above. The next step is sorting the data, i.e. the records of the list, in the growing order of the time of particle creation. Further, for time changing with $\mathrm{dt}$, all particles existing in time $t i=t 0+i d t$ are found and the information about them, i.e. their type and coordinates $(\mathrm{x}, \mathrm{y}, \mathrm{z})$, is recorded to support a file.

In the next step, the visualization program reads the data for time $t$, prepares the picture and updates the screen. Conducting this procedure step by step, animation of the EAS development is obtained. For the conducted visualization, we used the tools of Microsoft Visual $\mathrm{C}++2005$ and OpenGl as an interface API [8].

\section{The Visualization of the EAS}

The visualization was conducted in several variations. The basic alternatives are three forms of showing the current positions: as points on a scaled diagram with the coordinates X-Y-Z (illustrated in Fig. 2 and 3), as trajectories of all particles of the EAS in these coordinates (Fig. 4 and 5) and as movement trajectories of the particles existing in the actually shown time (Fig. 6 and 7). Furthermore, each of these visualization forms can be watched in the following ways: as a $3 \mathrm{D} \mathrm{X}-\mathrm{Y}-\mathrm{Z}$, in $2 \mathrm{D}$ plains $\mathrm{X}-\mathrm{Z}$ and $\mathrm{Y}-\mathrm{Z}$ as well as a $2 \mathrm{D} \mathrm{X}-\mathrm{Y}$ plain, which is the view from the bottom depicting the EAS falling from the atmosphere above. Each figure contains an additional plot illustrating the number of particles for the current altitude in the atmosphere. The current altitude in meters is placed in the right bottom containing the description.

\section{The Estimation of the Parameters $X_{-}$max and $R_{-}$font}

Having the above data, we created a program for reproducing the history of the EAS. For each simulated shower, it is possible to trace the history of the changes in the number of particles with the altitude in the atmosphere. This, in turn, allows finding the altitude with the maximum of the EAS development, $\mathrm{X}_{-}$max. This is a 
Pobrane z czasopisma Annales AI- Informatica http://ai.annales.umcs.pl

Data: 26/04/2023 14:24:22

$90 \quad$ Visualization of the extensive air shower and estimation of its parameters

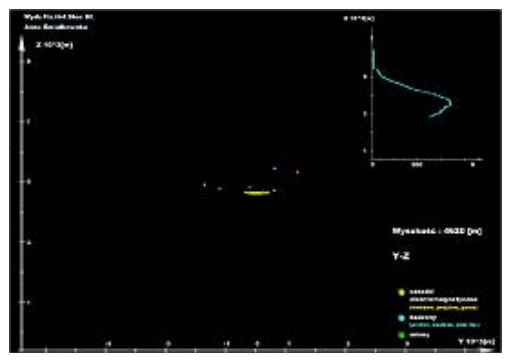

Fig. 2. Visualised particles as points in the $\mathrm{Y}-\mathrm{Z}$ scaled diagram.

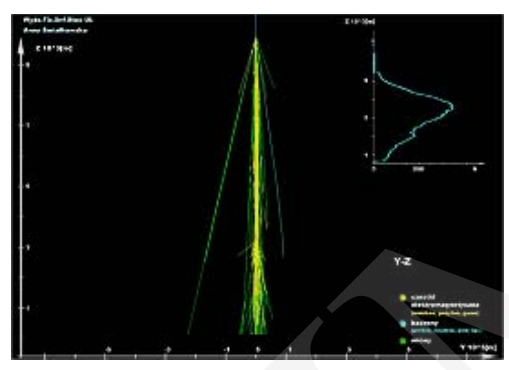

Fig. 4. Visualised trajectories of all particles of the EAS in the Y-Z scal.

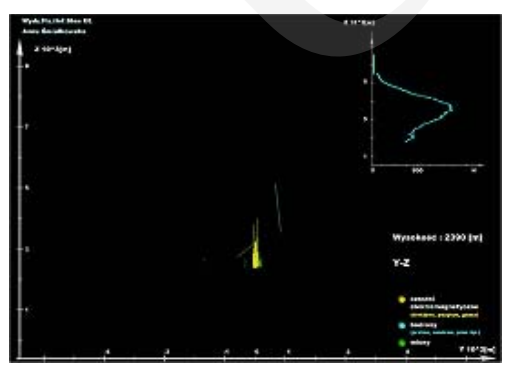

Fig. 6. Visualised movement trajectories of particles existing in the actually shown time of the EAS in the Y-Z scaled diagram.

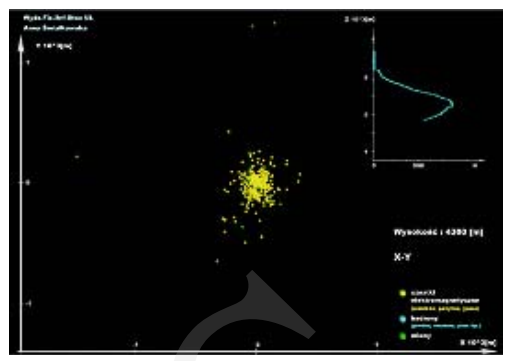

Fig. 3. Visualised particles as points in the X-Y scaled diagram.

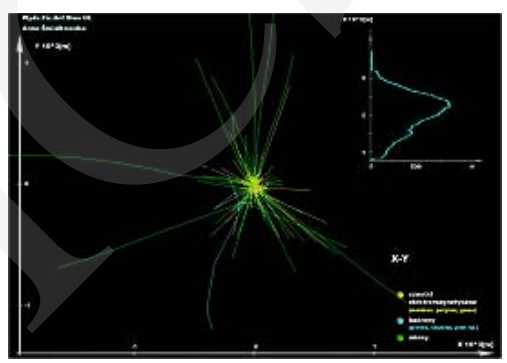

Fig. 5. Visualised trajectories of all particles of the EAS in the X-Y scaled diagram.

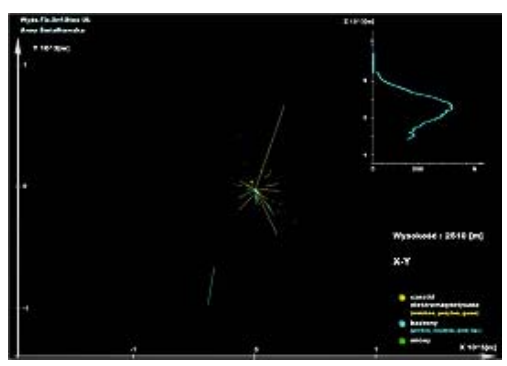

Fig. 7. Visualised movement trajectories of particles existing in the actually shown time of the EAS in the Y-Z scaled diagram.

very important contribution of our paper since X_max is the key parameter in the research of the EAS. 
Having the information about the time and place of the beginning and the end of each particle history, we can assess its speed (always close to c) and, further, the positions for any chosen time. We find that the fastest particles create a front forming a part of the sphere. Our program allows selecting data necessary for finding the curvature angle of the EAS front. For a chosen time interval dt, we record the data about the fastest particles. It corresponds to the choice of particles for a given altitude. The particles selected in this way come from the front of the EAS. The parameter R_front assessed from the data is the angle of the sphere describing the EAS front. The value of the parameter $\mathrm{R}$ _front is found as a circle function fit of the data. The fit has been made using the libraries ROOT or GSL. An example illustrating a fit conducted with ROOT is shown in Fig. 8.

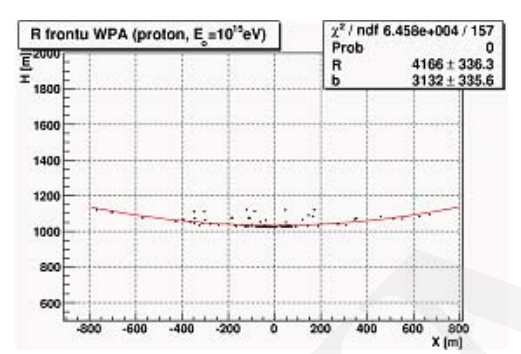

Fig. 8. An example illustrating a circle function fit conducted with ROOT.

The user version of our program constructed for physicists does not include visualization. When run for large datasets, i.e. for a lot of EAS, it can produce a series of the parameters described above. This is a great advantage for further research. It needs to be noted though that each case of filtration has to be checked in the graphs since it might occur that some pieces of data are chosen from the EAS front in a suboptimal way.

Our calculations show that both parameters estimated by the program have significantly different values, depending on the type of particle coming to the atmosphere from the outer space and initiating the EAS. In former papers [9], we presented an Artificial Neural Network for identifying the type of a primary particle depending on the parameters $\mathrm{X} \_$max and $\mathrm{R}$ _front.

Such tools are an important instrument for the research in physics on cosmic ray as well as in astrophysics.

\section{References}

[1] Hayakawa S., Cosmic Ray Physics (John Wiley \& Sons, New York, London, 1969, Mir, Moskwa, 1973): 547-630.

[2] Heck D. et al., Report FZKA 6019 (Forschungszentrum Karlsruhe, Karlsruhe, 1998).

[3] Dawson B. et al., The hybrid aperture and precision of the Auger observatory, ICRC Proc. (2001): 714-717. http://www.auger.org/news/PRagn/releases /AUGER_press_release_polish.pdf. 
[4] Yamamoto T. et al., Signatures of ultra-high energy cosmic ray composition from propagation of nuclei in intergalactic photon fields, Astropart. Phys. 20 (2004): 405-412, http://www-akeno.icrr.u-tokyo.ac.jp/AGASA/.

[5] http://www.cosmic-ray.org/.

[6] http://root.cern.ch.

[7] http://www.gnu.org/software/gsl/.

[8] Wright R. S. jr, Sweet M., OpenGL - Ksiega eksperta (Helion, Gliwice, 2005).

[9] Gustek G., The possibility of using artificial neural networks for the estimation of mass composition of high-energy primary cosmic ray, MCS thesis, University of Łódź (unpublished, 2008, in Polish). 